\title{
MORE PROPERTIES INHERITED BY THE LOWER RADICAL
}

\author{
R. F. ROSSA
}

\begin{abstract}
ABSTRACr. Various properties possessed by a class $M$ of not necessarily associative rings are inherited by the lower radical $L M$, including heredity to one-sided ideals or subrings, failure to contain nonzero noetherian rings and, in the associative case, failure to contain nonzero artinian rings.
\end{abstract}

Let $W$ be a universal class of (not necessarily associative) rings, that is, a class with the property that subrings and homomorphic images of rings in $W$ are again in $W$. A class of rings is said to be hereditary if every ideal of each ring in the class is also in the class. Let $M \subseteq W$; several constructions have been given for the Kurosh lower radical class $L M$ determined in $W$ by $M$. In [3], Leavitt and Hoffman used the construction of Anderson, Divinsky and Suliński [2] to prove that if $M$ is hereditary, then $L M$ is also hereditary. In this paper we employ the construction of Tangeman and Kreiling [4] to show that certain properties which may be possessed by $M$ are inherited by $L M$. This construction may be described briefly as follows. Let $M_{1}$ be the homomorphic closure of $M$. If $\beta>1$ is not a limit ordinal, admit a ring $R$ to $M_{\beta}$ if and only if there exists an ideal $I$ of $R$ such that $I$ and $R / I$ are both in $M_{\beta-1}$. If $\beta$ is a limit ordinal, let $R \in M_{\beta}$ iff $R$ is the union of a chain $\left\{I_{\gamma}: \gamma \in \Gamma\right\}$ of ideals of $R$ contained in $\bigcup_{\alpha<\beta} M_{\alpha}$. Then $L M=$ $\bigcup M_{\beta}$. Each of the classes $M_{\beta}$ is homomorphically closed [4, Lemma 2].

For the proof of Theorem 4 we require the following characterization of radical classes due to Amitsur.

THEOREM 1 [1]. The class $P \subseteq W$ is a radical class if and only if it satisfies the following three conditions.

(1) $P$ is homomorphically closed.

(2) $P$ is extension closed, i.e., if $I$ and $R / I$ are $P$-rings, then $R$ is also a P-ring.

(3) If $\left\{I_{\gamma}: \gamma \in \Gamma\right\}$ is a chain of $P$-ideals of $R \in W$, then $\bigcup_{\gamma} I_{\gamma}$ is a P-ideal of $R$.

Received by the editors September 13, 1971.

AMS 1970 subjec! classifications. Primary 13A10, 16A21; Secondary 16A46, 17A99.

Key words and phrases. Radical classes, hereditary radicals, lower radicals.

${ }^{1}$ Some of this work was done while the author was an NSF research participant at the University of Oklahoma.

(c) American Mathematical Society 1972 
A class $M$ of rings will be said to be right hereditary if every right ideal of each ring in $M$ is also in $M$; left and subring heredity are defined analogously. Tangeman and Kreiling using their construction gave a new proof that heredity in $M$ implies heredity in $L M$. With slight modifications in their proof [4, Theorem 3] it is easy to obtain the following.

THEOREM 2. If $M$ is right [left, subring] hereditary, then LM is also.

This result leads to a construction for a smallest right hereditary radical class in $W$ containing a given class $M$. Let $G_{1}=M$ and, for $n \geqq 2, G_{n}=$ $\left\{R \in W: R\right.$ is a right ideal of some ring in $\left.G_{n-1}\right\}$. Let $G(M)=\bigcup \bar{G}_{n}$.

THEOREM 3. $L G(M)$ is the unique minimal right hereditary radical class containing $M$.

Proof. $G(M)$ is clearly right hereditary so that $L G(M)$ is right hereditary by Theorem 2 . If $P$ is any right hereditary class in $W$ containing $M$, then $P$ must contain $G(M)$; if, moreover, $P$ is radical, then $P$ must contain $L G(M)$ as well by the minimality of the lower radical.

THEOREM 4. Let $M \subseteq W$ be a homomorphically closed class such that the center of every ring in $M$ is contained in a subring hereditary radical subclass $P$ of the class of commutative rings. Then the center of every ring in $L M$ is contained in $P$.

Proof. For each ring $R$, we let $Z(R)$ denote the center of $R$. We have assumed that every ring in $M_{1}=M$ has center in $P$. Thus suppose $\beta>1$ and that $Z(R) \in P$ for each $R \in \bigcup_{\alpha<\beta} M_{\alpha}$. Let $R \in M_{\beta}$. If $\beta$ is a limit ordinal then $R$ is the union of a chain $I_{\gamma}$ of its ideals contained in $\bigcup_{\alpha<\beta} M_{\alpha}$. Since $Z(R) \cap I_{\gamma} \subseteq Z\left(I_{\gamma}\right)$ for each $\gamma$ and $P$ is subring hereditary, $Z(R) \cap I_{\gamma} \in P$ for all $\gamma$. Thus $Z(R)=\bigcup\left(Z(R) \cap I_{\gamma}\right) \in P$ by Theorem 1 . If on the other hand $\beta-1$ exists, then $R$ has an ideal $J$ such that $J, R / J \in M_{\beta-1}$. Now $Z(R) /(Z(R) \cap J) \cong(Z(R)+J) / J \subseteq Z(R / J) \in P$ and $Z(R) \cap J \subseteq Z(J) \in P$ so that both $Z(R) / Z(R) \cap J$ and $Z(R) \cap J \in P$. By Theorem $1, Z(R) \in P$.

Thus, for example, if every ring in $M$ has zero center, then every ring in $L M$ has zero center. The class of rings with zero center is not homomorphically closed, so that the hypothesis that $M$ be homomorphically closed is essential in Theorem 4.

The next two results are concerned with classes of rings which lack one or the other of the chain conditions on left ideals. Although associativity is not required in Theorem 5, it appears to be essential in Theorem 6 .

THEOREM 5. Suppose $M$ is a homomorphically closed class containing no nonzero (left) noetherian rings. Then LM contains no nonzero noetherian rings. 
Proof. Again we proceed by induction. Suppose $R \in M_{\beta}$ is noetherian. In the nonlimit ordinal case, if $J, R / J \in M_{\beta-1}$, then $R / J$ is noetherian. Hence $R / J=0$ so that $R=J=0$ by the inductive hypothesis. In the limit ordinai case, if $R=\bigcup I_{\gamma}$, where $\left\{I_{\gamma}\right\}$ is a chain of ideals of $R$ contained in $\bigcup_{\alpha<\beta} M_{\alpha}$, then $\left\{I_{\gamma}\right\}$ musi have a maximal element $I_{0}$, forcing $R=I_{0}=0$.

THEOREM 6. SUPpose $M$ is a homomorphically closed class of associative rings containing no nonzero (left) artinian rings. Then $L M$ contains no nonzero artinian rings, where the lower radical is taken in the universal class of associative rings.

Proof. Suppose $R \in M_{\beta}$ is artinian. In the nonlimit ordinal case the proof is the same as in Theorem 5. Thus let $\beta$ be a limit ordinal and let $N$ denote the nil radical of $R . R$ is the union of a chain $\left\{I_{\gamma}\right\}$ of its ideals contained in $\bigcup_{\alpha<\beta} M_{\alpha}$. If $R / N \neq 0$, we have $R / N=\bigcup\left(I_{\gamma}+N\right) / N$ where each $\left(I_{\gamma}+N\right) / N \cong I /\left(I_{\gamma} \cap N\right) \in \bigcup_{\alpha<\beta} M_{\alpha}$. This is impossible since every ideal of a semisimple artinian ring is artinian. Thus $R=N$ and it is well known that the additive group of an artinian radical ring has the d.c.c. on subgroups. Therefore $R=0$ since otherwise each $I$ would have the d.c.c. on subgroups and hence on ideals.

The following example, due to W. G. Leavitt (private communication) shows that Theorem 6 does not hold for nonassociative rings in general. Let $R$ be the nonassociative algebra generated over the field $Q$ of rational numbers by the symbols $x_{1}, x_{2}, \cdots$ subject to the relations $x_{i} x_{j}=x_{j} x_{i}=x_{i}$ when $i<j, x_{i+1}^{2}=x_{i}$ for all $i \geqq 1$, and $x_{1}^{2}=0$. The ideals of $R$ are the principal ideals generated by $x_{i}, i \geqq 1$, so $R$ is artinian. Note that no nonzero homomorphic image of $Q x_{1}$ is artinian. However, since each nonzero homomorphic image of $R$ has an ideal isomorphic to $Q x_{1}$, we have $R \in L\left\{Q x_{1}\right\}$.

\section{REFERENCES}

1. S. A. Amitsur, A general theory of radicals. II. Radicals in rings and bicategories, Amer. J. Math. 76 (1954), 100-125. MR 15, 499.

2. R. Anderson, N. Divinsky and A. Suliński, Lower radical properties for associative and alternative rings, J. London Math. Soc. 41 (1966), 417-424. MR 33 \#4095.

3. A. E. Hoffman and W. G. Leavitt, Properties inherited by the lower radical, Portugal. Math. 27 (1968), 63-66. MR 41 \#6888.

4. D. Kreiling and R. L. Tangeman, Loxer radicals in nonassociative rings, J. Austral. Math. Soc. (to appear).

Division of Mathematics and Physics, Arkansas State University, State UniVERSITY, ARKANSAS 72467 